\title{
The use of real-time elastography in the assessment of gallbladder polyps: preliminary observations
}

\author{
Mehmet Akif Teber¹, Sinan Tan¹, Uğur Dönmez¹, Ali İpek¹, Ali Erkan Uçar², Halil Yıldırım¹, \\ Ahmet Aslan ${ }^{3}$, Halil Arslan 1
}

${ }^{1}$ Department of Radiology, ${ }^{2}$ Department of General Surgery, Atatürk Education and Research Hospital, Ankara, ${ }^{3}$ Department of Radiology, Bursa Şevket Yılmaz Education and Research Hospital Turkey

\begin{abstract}
Aims: Gallbladder polyps often have a benign nature. Current guidelines suggest surgical removal of polyps greater than $10 \mathrm{~mm}$. However, the accuracy of the size criteria is limited because neoplasia can be found in gallbladder polyps less than 10 $\mathrm{mm}$. The aim of this study was to evaluate the feasibility of real time elastography for gallbladder polyps and to demonstrate the elasticity properties of the polyps. Material and method: Fifty-three polypoid lesions of the gallbladder were prospectively examined with real-time elastography. Of these patients, 52 had a diagnosis of benign gallbladder polyps and one patient was accepted as a gallbladder carcinoma due to its clinical and radiological findings. B-mode and real-time elastographic images were simultaneously presented as a two-panel image, and the elastogram was displayed in a color scale that ranged from red (greatest strain, softest component), to green (average strain, intermediate component), to blue (no strain, hardest component). Results: The mean size for benign gallbladder polyps was $7.2 \pm 3 \mathrm{~mm}$ (range, 5-21 mm). All benign gallbladder polyps on consecutive real-time elastographic images appeared as having a high-strain elastographic pattern. Only one patient who was accepted with gallbladder carcinoma had a gallbladder polyp with low elasticity properties. Conclusions: Our study showed that real time elastography of gallbladder polyps is feasible. This novel approach may be useful for the characterization of polypoid lesions of the gallbladder.
\end{abstract}

Keywords: elastography, gallbladder, polyp, neoplasm

\section{Introduction}

Real-time elastography is a dynamic technique that was developed to evaluate tissue elasticity objectively by measuring the degree of distortion (strain) under external force. It presents data in color-coded displays on top of the gray-scale sonographic image [1]. Sonoelastography has been used as a diagnostic marker in clinical practice for different tissue types, including breast, thyroid gland, prostate, kidney, and liver [2-4]. To our knowledge, however, there are no published reports of real-time elastography used for gallbladder polyps.

Received 27.04.2014 Accepted 19.07.2014

Med Ultrason

2014, Vol. 16, No 4, 304-308

Corresponding author: Sinan Tan MD,

Ankara Atatürk Education and Research

Hospital, Department of Radiology

06400 Çankaya, Ankara, Turkey.

Phone : 05053148308

Email: drsinantan@gmail.com
Gallbladder polyps affect approximately $4-5 \%$ of the adult population and are often detected incidentally by sonography. The majority of gallbladder polyps are benign and most commonly include cholesterol polyps or inflammatory polyps $[5,6]$. In rare cases, these lesions may be neoplastic, and sometimes share the same appearance as benign polyps on sonographic examination [7]. Current guidelines suggest surgical removal of polyps greater than $10 \mathrm{~mm}$ and follow-up with subsequent sonography for polyps smaller than $10 \mathrm{~mm}[5,6]$. However, the accuracy of the size criteria is limited because the gallbladder neoplastic polyps are sometimes found less than $10 \mathrm{~mm}$ [8]. Therefore, a novel diagnostic approach based on imaging characteristics other than size criteria is needed to guide decisions on whether to perform cholecystectomy. Because malignant tissues are usually harder, or less elastic, than benign tissues, we hypothesized that assessment of tissue elasticity might be useful for gallbladder polyp characterization [2-4]. The purpose of this study was to evaluate the feasibility of sonoelastog- 
raphy in gallbladder polyps and to illustrate the elasticity properties of polyps.

\section{Materials and methods}

\section{Patients}

This prospective observational study was approved by the institutional review board, and informed consent was obtained from all of the reviewed subjects. Seventyfour patients underwent sonography, including elastography of gallbladder polyps, in our institution. A polyp was defined on sonography as a lesion attached to the gallbladder wall that was non-shadowing, non-mobile and hyperechoic to bile $[5,9]$. Patients were included in the study if they had any of the following: sonographic follow-up for at least 2 years, pathology report, or who were diagnosed on the basis of characteristic clinical and radiologic findings. Of these 74 patients, 21 were excluded due to insufficient follow-up or lack of a reference standard to establish diagnosis. The final cohort included 53 patients with gallbladder polyps (32 women and 21 men; mean $[ \pm \mathrm{SD}]$ age, $44.1 \pm 10.6$ years; range, $22-70$ years).

Of these patients, 52 had a diagnosis of benign gallbladder polyps. 40 patients with polyps (77\%) had a sonography follow-up. The remaining $12(23 \%)$ patients underwent cholecystectomy. In addition, we included one patient who had multiple metastatic lesions to the liver and lung with a polypoid lesion in the gallbladder, which was accepted as a gallbladder carcinoma.

Five patients had more than one polyp, and only the largest lesion was used for analysis; one of these patients had acromegaly.

\section{Equipment and Scanning}

Sonoelastography examinations were performed by one of two radiologists. These practitioners had 8-13 years of experience with conventional sonography and 2 years of experience in elastography. The patients underwent sonoelastographic examinations in the supine and left lateral decubitus positions during deep inspiration breath-hold after fasting for at least 6 hours, using a digital sonography scanner (Logiq E9, GE Healthcare, Milwaukee, Wisconsin, USA) with a convex $2.8-5-\mathrm{MHz}$ multifrequency transducer. B-mode and real-time elastographic images were simultaneously presented as a twopanel image, and the elastogram was displayed in a color scale that ranged from red (greatest strain, softest component), to green (average strain, intermediate component), to blue (no strain, hardest component) [10]. Elastography scans were performed according to a standard protocol as follows: after identification of a gallbladder polyp on a gray scale ultrasound image, real-time elastography was performed using the same probe while applying minimal pressure to the probe, since the gallbladder itself receives pressure from the heartbeat [11]; images were obtained only when optimal compression was in the 5-7-bar range [3]; the scanning protocol was completed after confirming that several stable and reproducible images depicted almost the same color map of the polyp; static and moving images were also recorded for later review; elasticity properties were decided in consensus by two radiologists.

\section{Statistical Analysis}

The statistical Package for Social Sciences (SPSS version 15.0 Chicago, IL, USA) was used for statistical analysis. The numeric variables were expressed as either mean \pm SD or number (percentage), where appropriate. Differences in the general distribution of elastographic patterns were evaluated by the Shapiro Wilk test. The Kruskal Wallis test was used for comparisons between elastographic patterns and gallbladder polyp size. Statistical significance was set as $\mathrm{p}<0.05$.

\section{Results}

The mean size for benign gallbladder polyps was $7.2 \pm 3 \mathrm{~mm}$ (range, $5-21 \mathrm{~mm}$ ). There were no statistically significant differences between elastographic patterns and gallbladder polyp size $(\mathrm{p}=0.64)$. The mean distance between the surface of the gallbladder polyps and the skin surface was $45.1 \pm 7.7 \mathrm{~mm}$ (range, 27-60 mm).

Real-time elastographic gallbladder polyp images obtained from the 53 patients were interpretable for assessing the elastographic pattern. All benign gallbladder polyps on consecutive real-time elastographic images appeared as having a greater than average or average elastographic pattern. Elastographic images showed most gallbladder polyps as a mosaic of green, yellow and red (28 out of $52,53.8 \%$ ), whereas the remaining polyps showed either entirely green (12 out of 52, 23.1\%) or red with partially yellow areas (12 out of 52, 23.1\%) (fig 1-4). The histopathologic subtype was found to be cholesterol polyps in 9 cases and hyperplastic polyps in 3 cases that underwent cholecystectomy in 12 patients. Elastographic images of all hyperplastic polyps and 5 of the cholesterol polyps were a mosaic of green, yellow and red, 2 of the cholesterol polyps were entirely green and 2 of the cholesterol polyps were red with partially yellow areas.

In our study, only one patient had a gallbladder polyp with low elasticity properties. The mean size for this polyp was $19 \mathrm{~mm}$. The distance between the surface of the polyp and the skin surface was $41 \mathrm{~mm}$. In this case, the elastogram showed a predominantly stiff (blue) appearance within the polypoid lesion, with minimal soft tissue areas (ranging from red to green) (fig 5). In this case, there were multiple metastatic lesions, and the patient died 10 days after sonographic examination. Al- 


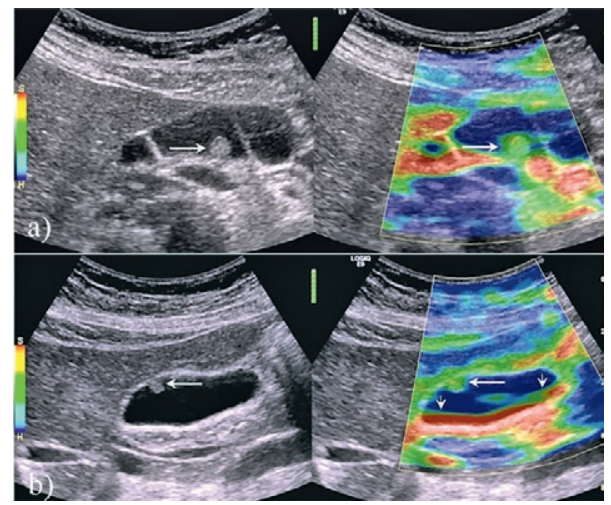

Fig 1. Gray-scale and color examples of high-strain elastographic patterns that are entirely green on the elastogram: a) 40-year-old woman with gallbladder polyp (arrow) identified as benign on follow-up sonography; b) a 43-year-old man with a gallbladder polyp (long arrow) identified as benign on follow-up sonography. Small arrows denote a three-layered artifact pattern.

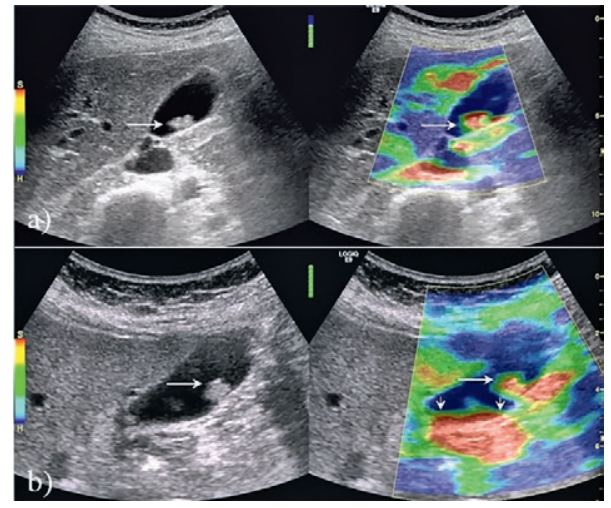

Fig 2. Gray-scale and color examples of high-strain elastographic patterns with a mosaic of green, yellow and red on the elastogram: a) 34-year-old woman with gallbladder polyp (arrow) evaluated as a cholesterol polyp in pathologic examination; b) a 47-year-old woman with a gallbladder polyp (long arrow) evaluated as a cholesterol polyp in pathologic examination. Small arrow denotes a three-layered artifact pattern.

though the histopathologic diagnosis in this case could not be confirmed, the polypoid lesion was evaluated as a gallbladder carcinoma based on clinical and radiological findings.

\section{Discussions}

Real-time elastography measures the degree of elasticity distribution within tissues under compression and is based on the principle that the softer parts of tissues deform more easily than the harder parts. Because many diseases correlate with changes in the mechanical properties of tissues, this technique has been successfully

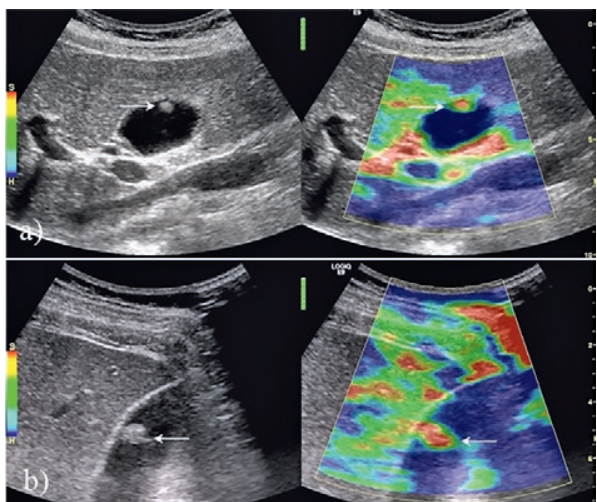

Fig 3. Gray-scale and color examples of high-strain elastographic patterns with red and/or yellow on the elastogram: a) 45-year-old man with gallbladder polyp (arrow) identified as benign on follow-up sonography; b) a 42-year-old woman with gallbladder polyp identified as benign on follow-up sonography. A three-layered artifact pattern is seen around the polyp.

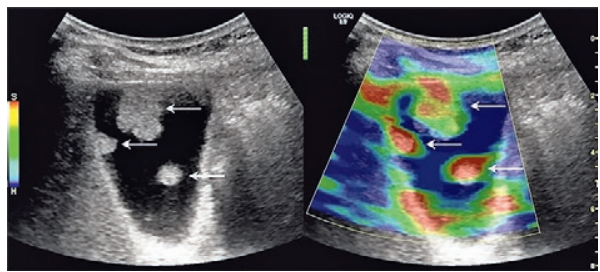

Fig 4. A 50-year-old woman who was diagnosed with acromegaly due to a pituitary adenoma. The patient had multiple large gallbladder polyps, but refused surgery. Sonography follow-up over 2 years was performed, and the size of the polyps remained stable. Arrows denote polyps with a high-strain elastographic pattern on the elastogram. A three-layered artifact pattern is seen around the polyps.

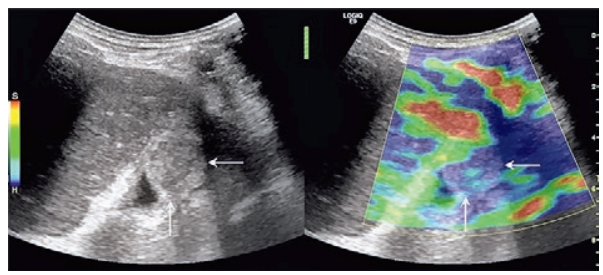

Fig 5. A 58-year-old man with lung and liver metastasis was evaluated with carcinoma from clinical and radiologic findings. Gray-scale and color examples of a low-strain elastographic pattern. Polypoid lesion (arrows) appears almost entirely stiff (blue, with some red and yellow) on the elastogram.

applied in detecting and assessing various pathologies of thyroid gland, breast, lymph nodes, major salivary gland, prostate, kidney, and liver [1-3,11-17]. However, to our knowledge, no study has been reported previously in the medical literature using real time sonoelastography to assess gallbladder polyps. This study revealed 
that sonoelastography of gallbladder polyps is a feasible method.

With the increased use of sonography, gallbladder polyps are often detected incidentally. Although most polyps are benign in nature, some early carcinomas show a similar appearance to benign polyps. Sonography has been used extensively in the pre-operative management of polyps, but this technique is generally not sufficient to differentiate between benign and malignant polyps, with the exception of advanced gallbladder cancer $[18,19]$. On the other hand, the overall prognosis for gallbladder cancer is extremely poor, with less than a 10\% 5- year survival rate $[6,20]$. Current guidelines in the management of gallbladder polyps are generally established on the basis of polyp size, which recommend sonography follow-up for polyps smaller than $10 \mathrm{~mm}$ and surgical intervention for those 10 $\mathrm{mm}$ or greater [5]. However, this strategy may well produce increased patient anxiety and unnecessary follow-up examinations [6]. Therefore, a novel diagnostic approach based on polyp imaging findings other than the size criteria can help in polyp characterization. Newer sonographic techniques such as contrast enhanced US (CEUS) gave promising results on differentiating benign and malignant gallbladder lesions [21]. But administration of contrast agent, detection of the contrast enhancement pattern or wash-out phenomenon of the contrast agent, the need for perfusion quantification parameters, and application of transducer in a stable position for at least 3 minutes may limit the use of CEUS [17,21]. We think that elastography can be useful in the characterization of gallbladder polyps as a new technique, although there is insufficient data at present for this hypothesis. In this study, we obtained interpretable images from all of the gallbladder polyps of various sizes. In relation to other studies on differentiating benign lesions, all benign gallbladder polyps were characterized as having high elasticity properties. Only one of the polyps in this study was interpreted as having a low elasticity on the elastogram. Although the histopathologic diagnosis could not be confirmed, it was felt that the patient had the diagnostic features of a gallbladder carcinoma. In addition, we found in the majority of cases threelayered areas (blue, green, and red) on the elastogram, which were usually observed in the dependent portion of the gallbladder, and/or around the polyp. This appearance is an artifact of elastography due to liquid in the lumen of the gallbladder [12].

Our study had several limitations. First, the current study had only one case showing features of a malignant polyp, and pathologic correlation of the case was not provided. Second, most of the gallbladder polyps were accepted as benign by sonographic follow-up without histopathological diagnosis. Third, elastography itself has certain limitations, including relatively high operator dependency, factors affecting the performance of elastography, such as size, depth, and density of the lesion, strain values containing variables with different degrees of compression and solid components affected by the lack of strain of the fluid portion [2,3,14,22]. Finally, description of the elasticity pattern is a subjective method. Nevertheless, a quantitative analysis of tissue elasticity of gallbladder polyps might be useful for providing measurable thresholds.

In conclusion, real time elastography is feasible in patients with gallbladder polyps. In our study, all benign gallbladder polyps were evaluated as having a high-strain elastographic pattern. Due to the above-mentioned limitations, however, we do not claim from the available data to have provided a full clinical investigation demonstrating the efficacy of elastography in the differential diagnosis of gallbladder polyps. To evaluate the diagnostic performance of this method in gallbladder polyps, larger-scale studies must be performed that include patients diagnosed as having malignant polyps, along with ideal histopathologic analysis.

Acknowledgments: We gratefully acknowledge the assistance of Dr Fatih Tufan for his constructive criticism and comments during the writing of this paper.

\section{Conflict of interest: none}

\section{References}

1. Ciledag N, Arda K, Aribas BK, Aktas E, Köse SK. The utility of ultrasound elastography and MicroPure imaging in the differentiation of benign and malignant thyroid nodules. AJR Am J Roentgenol 2012; 198: W244-W249.

2. Itoh A, Ueno E, Tohno E, et al. Breast disease: clinical application of US elastography for diagnosis. Radiology 2006; 239: 341-350.

3. Tan S, Özcan MF, Tezcan F, et al. Real-time elastography for distinguishing angiomyolipoma from renal cell carcinoma: preliminary observations. AJR Am J Roentgenol 2013; 200: W369-W375.

4. Moon HJ, Sung JM, Kim EK, Yoon JH, Youk JH, Kwak J. Diagnostic performance of gray-scale US and elastography in solid thyroid nodules. Radiology 2012; 262: 1002-1013.

5. Pedersen MR, Dam C, Rafaelsen SR. Ultrasound follow-up for gallbladder polyps less than $6 \mathrm{~mm}$ may not be necessary. Dan Med J 2012;59:A4503.

6. Corwin MT, Siewert B, Sheiman RG, Kane RA. Incidentally detected gallbladder polyps: is follow-up necessary?Long-term clinical and US analysis of 346 patients. Radiology 2011; 258: 277-282.

7. Lee KF, Wong J, Li JC, Lai PB. Polypoid lesions of the gallbladder. Am J Surg 2004; 188: 186-190. 
8. Terzi C, Sökmen S, Seçkin S, Albayrak L, Uğurlu M. Polypoid lesions of the gallbladder: report of 100 cases with special reference to operative indications. Surgery 2000; 127: 622-627.

9. Lin WR, Lin DY, Tai DI, et al. Prevalence of and risk factors for gallbladder polyps detected by ultrasonography among healthy Chinese: analysis of 34669 cases. J Gastroenterol Hepatol 2008; 23: 965-969.

10. Cho N, Jang M, Lyou CY, Park JS, Choi HY, Moon WK. Distinguishing benign from malignant masses at breast US: combined US elastography and color Doppler US--influence on radiologist accuracy. Radiology 2012; 262: 80-90.

11. Koizumi Y, Hirooka M, Kisaka Y, et al. Liver fibrosis in patients with chronic hepatitis $\mathrm{C}$ : noninvasive diagnosis by means of real-time tissue elastography-establishment of the method for measurement. Radiology 2011; 258 :610-617.

12. Satake H, Nishio A, Ikeda M, et al. Predictive value for malignancy of suspicious breast masses of BI-RADS categories 4 and 5 using ultrasound elastography and MR diffusionweighted imaging. AJR Am J Roentgenol 2011; 196: 202-209.

13. Alam F, Naito K, Horiguchi J, Fukuda H, Tachikake T, Ito K. Accuracy of sonographic elastography in the differential diagnosis of enlarged cervical lymph nodes: comparison with conventional B mode sonography. AJR Am J Roentgenol 2008; 191: 604-610.

14. Dumitriu D, Dudea S, Botar-Jid C, Baciuţ M, Baciuţ G. Real-time sonoelastography of major salivary gland tumors. Am J Roentgenol 2011 ;197: W924-W930.
15. Celebi I, Mahmutoglu AS. Early results of real-time qualitative sonoelastography in the evaluation of parotid gland masses: a study with histopathological correlation. Acta Radiol 2013; 54: 35-41.

16. Navarro B, Úbeda B, Vallespí M, Wolf C, Casas L, Browne JL. Role of elastography in the assessment of breast lesions: Preliminary results. J Ultrasound Med 2011; 30: 313-321.

17. Fodor D, Pascu I, Pop S, Poanta L. The utility of elastography and CEUS for the differentiation between benign and malignant cervical lymphadenopaty. Three cases report. Med Ultrason 2013; 15: 63-66.

18. Andren-Sandberg A. Diagnosis and management of gallbladder polyps. N Am J Med Sci 2012; 4: 203-211.

19. Zielinski MD, Atwell TD, Davis PW, Kendrick ML, Que FG. Comparison of surgically resected polypoid lesions of the gallbladder to their pre-operative ultrasound characteristics. J Gastrointest Surg 2009; 13: 19-25.

20. Hussain HM, Little MD, Wei S. AIRP best cases in radiologic-pathologic correlation: gallbladder carcinoma with direct invasion of the liver. Radiographics 2013; 33: 103108 .

21. Sparchez Z, Radu P. Role of CEUS in the diagnosis of gallbladder disease. Med Ultrason 2012; 14: 326-330.

22. Moon HJ, Sung JM, Kim EK, Yoon JH, Youk JH, Kwak JY. Diagnostic performance of gray-scale US and elastography in solid thyroid nodules. Radiology 2012; 262: 1002-1013. 